\title{
Adult hippocampal neurogenesis and plasticity in the infrapyramidal bundle of the mossy fiber projection: II. Genetic covariation and identification of Nos1 as linking candidate gene
}

\section{Julia Krebs ${ }^{1+\ddagger}$, Benedikt Römer $^{1+\ddagger}$, Rupert W. Overall ${ }^{1}, K_{\text {Klaus Fabel }}{ }^{1,2}$, Harish Babu $^{1 \dagger}$, Moritz D. Brandt $^{1}$, Robert W. Williams ${ }^{3}$, Sebastian Jessberger ${ }^{4}$ and Gerd Kempermann ${ }^{1,2 *}$}

' Genomics of Regeneration, Center for Regenerative Therapies Dresden, Dresden, Germany

2 German Center for Neurodegenerative Diseases, Dresden, Germany

${ }^{3}$ Department of Anatomy and Neurobiology, University of Tennessee Health Science Center, Memphis, TN, USA

${ }^{4}$ Department of Biology, Institute of Cell Biology, Eidgenössische Technische Hochschule Zürich, Zürich, Switzerland

\section{Edited by:}

Steven A. Goldman, University of Rochester Medical Center, USA

Reviewed by:

Angelique Bordey, Yale University School of Medicine, USA

Brian R. Christie, University of

Victoria, Canada

\section{${ }^{*}$ Correspondence:}

Sebastian Jessberger, Department of Biology, Institute of Cell Biology, Eidgenössische Technische

Hochschule Zürich, Schafmattstr. 18, 8093 Zürich, Switzerland.

e-mail: jessberger@cell.biol.ethz.ch, Gerd Kempermann, DFG Research

Center for Regenerative Therapies

Dresden, Center for Regenerative

Therapies Dresden, and DZNE,

German Center for

Neurodegenerative Diseases,

Dresden, Tatzberg 47-49, 01307

Dresden, Germany.

e-mail:gerd.kempermann@

crt-dresden.de

${ }^{t}$ Present address:

Julia Krebs, Psychiatric Hospital of the University of Basel, Basel,

Switzerland:

Benedikt Römer, Department of

Psychiatry, Stavanger University

Hospital, Stavanger, Norway:

Harish Babu, Department of

Neurosurgery, Stanford University,

Stanford, CA, USA.

¥ Julia Krebs and Benedikt Römer

have contributed equally to this work.
The hippocampus of adult rodents harbors two systems exhibiting structural plasticity beyond the level of synapses and dendrites. First, the persistent generation of granule cells (adult neurogenesis); second, dynamic changes in the mossy fibers (MF), in particular in the infrapyramidal mossy fiber (IMF) tract. Because MFs are the axons of granule cells, the question arises whether these two types of plasticity are linked. In the first part of this study (Römer et al., 2011) we have asked how both traits are regulated in relation to each other. In the present part, we asked whether, besides activity-dependent co-regulation, there would also be signs of genetic co-regulation and co-variance. For this purpose we used the BXD panel of recombinant inbred strains of mice, a unique genetic reference population that allows genetic association studies. In 31 BXD strains we did not find correlations between the traits describing the volume of the MF subfields and measures of adult neurogenesis. When we carried out quantitative trait locus mapping for these traits, we found that the map for IMF volume showed little overlap with the maps for the other parts of the projection or for adult neurogenesis, suggesting that to a large degree the IMF is regulated independently. The highest overlapping peak in the genome-wide association maps for IMF volume and the number of new neurons was on distal chromosome 5 (118.3-199.2 Mb) with an LRS score of 5.5 for IMF and 6.0 for new neurons. Within this interval we identified Nos1 (neuronal nitric oxide synthase) as a cis-acting (i.e., presumably autoregulatory) candidate gene. The expression of Nos 1 is has been previously linked with both IMF and adult neurogenesis, supporting our findings. Despite explaining on its own very little of the variance in the highly multigenic traits studied, our results suggest Nos1 may play a part in the complex genetic control of adult neurogenesis and IMF morphology.

Keywords: hippocampus, stem cell, learning, memory, quantitative trait locus

\section{INTRODUCTION}

Correlational analysis between behavior and anatomical variation can be a powerful tool to both develop and test hypotheses about relationships between structure and function. While correlation may not imply causation, the converse is almost always true: common causes should almost always lead to strong correlations. For this reason, correlation can be a powerful tool to test whether two processes share causes (Shipley, 2002). In the present study we exploit a correlational approach to dissect common causes of adult neuronal plasticity in the mouse hippocampus. Our source material consists of a large genetic reference panel of extraordinarily well studied strains of mice. These mice are also highly divergent in terms of levels of adult neurogenesis (Kempermann et al., 2006) that is the lifelong production of new granule cells in the adult dentate gyrus. This natural perturbation of adult neurogenesis levels is an excellent platform with which to test 
common cellular and molecular mechanisms that underlie brain plasticity.

The size of the infrapyramidal mossy fiber (IMF) projection, which is formed by dentate granule cell axons projecting to pyramidal cells in region CA3, correlates positively with performance in a variety of behavioral tasks (Schwegler et al., 1981, 1988, 1990; Lipp et al., 1984; Crusio et al., 1987; Roullet and Lassalle, 1990; Schopke et al., 1991; Bernasconi-Guastalla et al., 1994; Laghmouch et al., 1997); for a review see: Crusio and Schwegler (2005). In contrast, the suprapyramidal mossy fiber (SMF) projection presents the majority of the connecting fibers and is much more stable than the immensely plastic IMF (Schwegler et al., 1981; Crusio et al., 1989). Nevertheless, the underlying mechanism that determine, why a relatively larger IMF would positively correlate with hippocampal function have so far remained unidentified. One proposed explanation has been that IMF and SMF connect to different sets of dendrites (Blackstad et al., 1970) so that variations in the distribution of mossy fibers (MF) might affect how granule cells drive pyramidal cells in CA3 (Gonzales et al., 2001).

Size of the MF connection is a classical "quantitative trait," in that it varies continuously between individuals (and inbred strains). A few studies have consequently explored genetic variation of this trait (Nowakowski, 1984; Lassalle et al., 1999; Crusio et al., 2007). Whereas the genetic variation of the trait itself was unambiguously confirmed, the studies did not lead to the identification of clear candidate genes.

The neuroanatomical plasticity of dentate gyrus-CA3 connections, however, might represent more than just structural reorganization of pre-existing circuits given the birth of new neurons in the dentate gyrus throughout adulthood. New neurons send axons toward CA3 and form functional synapses with postsynaptic targets (Zhao et al., 2006; Toni et al., 2008). Therefore, we asked whether adult neurogenesis and size of the IMF as two different forms of structural plasticity in the adult hippocampus might be related.

In the first part of the present study, we had explored, whether MF plasticity in response to extrinsic stimuli would show coregulation with adult neurogenesis. We had shown that new neurons contribute to the highly plastic IMF projection and that both are co-regulated by environmental enrichment and seizure activity (Römer et al., 2011).

In the present, second part we turn to a potentially shared underlying genetics. The MF traits were analyzed in the genetic reference population of the BXD set of recombinant inbred (RI) strains of mice, in which we had previously studied adult hippocampal neurogenesis (Kempermann et al., 2006). RI mice are essentially inbred brother-sister matings from the F2 generation of an initial cross between the two name-giving parental strains of the set, here C57BL/6J (B) and DBA2/J (D). Due to the nature of the breeding scheme, the individual RI strains represent roughly equal mixes of the parental genome but the pattern is unique to each strain. All strains of the BXD set are fully genotyped based on SNPs; the parental strains have been sequenced. The genetic information as well as numerous published phenotypes (including those of the present study) are deposited and publicly accessible at www.genenetwork.org.

\section{MATERIALS AND METHODS ANIMALS}

This study used a total of 94 female mice from 29 strains of the BXD RI panel (Taylor, 1978; Taylor et al., 1999) as well as the parentals, C57BL/6J and DBA/2J. The average age of the animals was $71 \pm 1.2$ days old. The data on adult neurogenesis from these animals have been published previously (Kempermann et al., 2006).

Correlational studies were done in RI strains of mice from the RI strains from the set BXD based on C57BL/6J (B) and $\mathrm{DBA} / 2 \mathrm{~J}$ (D). Data on adult neurogenesis have been published
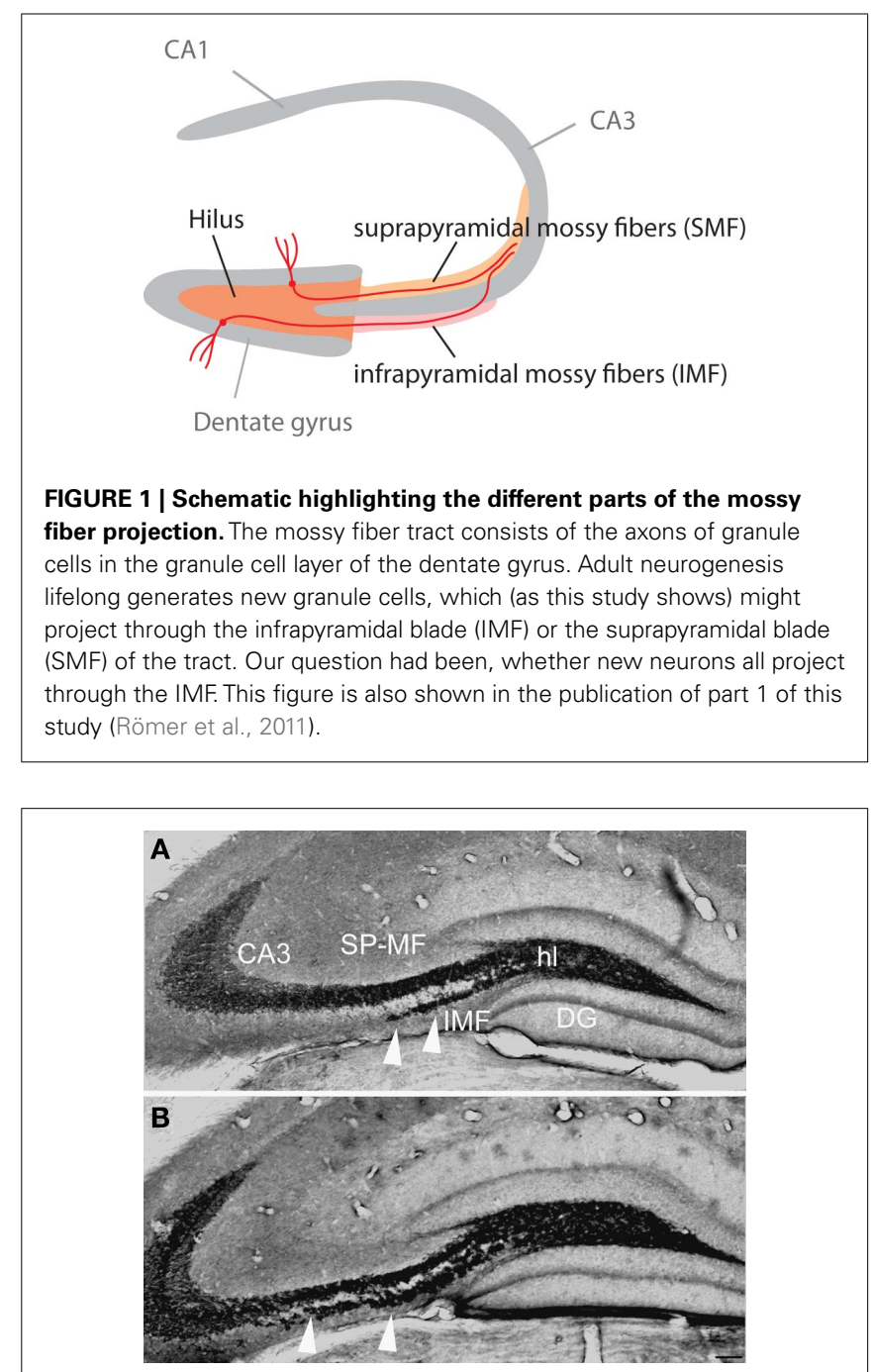

FIGURE 2 | Strain differences in the size of the mossy fiber subfields. $(\mathbf{A}, \mathbf{B})$ Besides differences in the rate of neurogenesis, the parental strains of the recombinant inbred set C57BL/6J (B) and DBA/2J (A) showed differences in mossy fiber morphology (anti-synaptoporin staining). C57BL/6J mice had a larger IMF [arrowheads in $(\mathbf{A}, \mathbf{B})$ ] and a larger hilus (hl) compared to DBA/2J mice, whereas the suprapyramidal mossy fiber tract tended to be bigger in DBA/2J mice. Scale bar in (B): $100 \mu \mathrm{m}$. DG, dentate gyrus; CA3, cornu ammonis area 3; SMF, suprapyramidal mossy fibers; IMF, infrapyramidal mossy fibers. 
previously (Kempermann et al., 2006). The extent of the MF projection was measured in tissue from these animals as described below.

\section{MORPHOMETRIC ANALYSIS}

The size of the hippocampal MF projections and terminal fields were revealed by an immunohistochemical staining procedure against the presynaptic vesicle protein synaptoporin. Due to its high content of synaptoporin, the MF tract can be reliably visualized using antibodies against synaptoporin (Singec et al., 2002). Every sixth section was analyzed to assess the size of the hilus, the suprapyramidal mossy fiber terminal fields (SMF), and the IMF (Figure 1). A semi-automated morphometric system (Stereoinvestigator, Microbrightfield, Magdeburg) consisting of a CCD camera (Hitachi) connected to a conventional light microscope (Leica DM-RXE) and a personal computer were used. MF fields were outlined from the projected image (magnification: $10 \times$, NA: 0.30 ) and area sizes were determined using the area measurement tool (based on the Cavalieri estimator) of the stereology software Stereo
Investigator (MicroBrightField). To obtain the volume of the terminal fields, the sum of areas measured was multiplied by the inverse of the sampling fraction (6) and 40 (the section thickness in micrometer).

\section{OTL MAPPING AND STATISTICAL ANALYSES}

All numerical baseline analyses were performed using Statview 5.0.1 for Macintosh or R. For all comparisons ANOVA was performed followed by Fisher's post hoc test, when appropriate. Differences were considered significantly different at a $p<0.05$. Data from the Lassalle et al. (1999) study (see Discussion) were obtained from www.genenetwork.org. All numbers in the text and tables are mean \pm SEM.

Correlations were calculated with the analysis tools at www.genenetwork.org.

\section{HERITABILITY}

An estimate of the broad-sense heritability was calculated as the variance of the strain means (the genetic variance) divided by

Table 1 | Volumes of the mossy fiber tract and its sub-structures in BXD strains.

\begin{tabular}{|c|c|c|c|c|c|c|c|c|c|c|}
\hline & \multicolumn{2}{|c|}{ Hilus } & \multicolumn{2}{|c|}{ SMF } & \multicolumn{2}{|c|}{ IMF } & \multicolumn{2}{|c|}{ Hilus + SMF } & \multicolumn{2}{|c|}{ MF (total) } \\
\hline & Mean & SE & Mean & SE & Mean & SE & Mean & SE & Mean & SE \\
\hline C57 & 0.1246 & 0.0135 & 0.1551 & 0.0207 & 0.0440 & 0.0032 & 0.2797 & 0.0295 & 0.3237 & 0.0325 \\
\hline DBA & 0.0904 & 0.0118 & 0.2173 & 0.0173 & 0.0197 & 0.0018 & 0.3077 & 0.0220 & 0.3274 & 0.0234 \\
\hline BXD1 & 0.1187 & 0.0167 & 0.2058 & 0.0192 & 0.0363 & 0.0070 & 0.3246 & 0.0318 & 0.3609 & 0.0387 \\
\hline BXD2 & 0.0929 & 0.0012 & 0.1583 & 0.0058 & 0.0422 & 0.0032 & 0.2512 & 0.0070 & 0.2934 & 0.0102 \\
\hline BXD5 & 0.1350 & 0.0483 & 0.1628 & 0.0145 & 0.0456 & 0.0090 & 0.2978 & 0.0628 & 0.3435 & 0.0718 \\
\hline BXD6 & 0.1081 & 0.0205 & 0.1892 & 0.0272 & 0.0236 & 0.0027 & 0.2974 & 0.0326 & 0.3210 & 0.0346 \\
\hline BXD9 & 0.1601 & 0.0357 & 0.1986 & 0.0417 & 0.0281 & 0.0035 & 0.3587 & 0.0740 & 0.3867 & 0.0774 \\
\hline BXD11 & 0.1181 & 0.0469 & 0.1106 & 0.0276 & 0.0208 & 0.0046 & 0.2287 & 0.0704 & 0.2495 & 0.0734 \\
\hline BXD12 & 0.0844 & 0.0066 & 0.1357 & 0.0065 & 0.0347 & 0.0008 & 0.2201 & 0.0127 & 0.2548 & 0.0127 \\
\hline BXD14 & 0.0889 & 0.0209 & 0.1616 & 0.0206 & 0.0214 & 0.0051 & 0.2505 & 0.0412 & 0.2719 & 0.0455 \\
\hline BXD15 & 0.0800 & 0.0115 & 0.1645 & 0.0244 & 0.0244 & 0.0040 & 0.2445 & 0.0345 & 0.2689 & 0.0384 \\
\hline BXD16 & 0.1330 & 0.0112 & 0.1903 & 0.0379 & 0.0380 & 0.0039 & 0.3233 & 0.0439 & 0.3613 & 0.0469 \\
\hline BXD18 & 0.1450 & 0.0234 & 0.1664 & 0.0041 & 0.0524 & 0.0118 & 0.3114 & 0.0275 & 0.3637 & 0.0393 \\
\hline BXD19 & 0.1323 & 0.0149 & 0.1968 & 0.0303 & 0.0285 & 0.0058 & 0.3291 & 0.0449 & 0.3576 & 0.0507 \\
\hline BXD21 & 0.0990 & 0.0070 & 0.1855 & 0.0019 & 0.0373 & 0.0009 & 0.2844 & 0.0089 & 0.3217 & 0.0080 \\
\hline BXD22 & 0.1051 & 0.0163 & 0.1850 & 0.0170 & 0.0253 & 0.0017 & 0.2901 & 0.0064 & 0.3155 & 0.0049 \\
\hline BXD24 & 0.0656 & 0.0171 & 0.1070 & 0.0256 & 0.0259 & 0.0053 & 0.1726 & 0.0426 & 0.1985 & 0.0476 \\
\hline BXD25 & 0.0642 & 0.0109 & 0.1500 & 0.0094 & 0.0214 & 0.0036 & 0.2142 & 0.0154 & 0.2356 & 0.0168 \\
\hline BXD27 & 0.0712 & 0.0161 & 0.1478 & 0.0190 & 0.0278 & 0.0038 & 0.2191 & 0.0334 & 0.2468 & 0.0365 \\
\hline BXD28 & 0.0652 & 0.0065 & 0.1116 & 0.0230 & 0.0223 & 0.0028 & 0.1768 & 0.0294 & 0.1991 & 0.0322 \\
\hline BXD29 & 0.0697 & 0.0114 & 0.1143 & 0.0063 & 0.0147 & 0.0026 & 0.1840 & 0.0062 & 0.1987 & 0.0088 \\
\hline BXD30 & 0.0842 & 0.0152 & 0.1181 & 0.0246 & 0.0284 & 0.0084 & 0.2023 & 0.0397 & 0.2307 & 0.0431 \\
\hline BXD31 & 0.0889 & 0.0027 & 0.1455 & 0.0194 & 0.0229 & 0.0042 & 0.2344 & 0.0195 & 0.2573 & 0.0217 \\
\hline BXD32 & 0.1004 & 0.0173 & 0.1302 & 0.0098 & 0.0214 & 0.0024 & 0.2306 & 0.0247 & 0.2520 & 0.0272 \\
\hline BXD33 & 0.0992 & 0.0231 & 0.1178 & 0.0223 & 0.0276 & 0.0055 & 0.2170 & 0.0454 & 0.2446 & 0.0509 \\
\hline BXD34 & 0.0865 & 0.0184 & 0.1136 & 0.0252 & 0.0364 & 0.0070 & 0.2000 & 0.0435 & 0.2364 & 0.0504 \\
\hline BXD35 & 0.1608 & 0.0200 & 0.1975 & 0.0022 & 0.0371 & 0.0006 & 0.3584 & 0.0221 & 0.3954 & 0.0227 \\
\hline BXD38 & 0.1144 & 0.0067 & 0.1585 & 0.0109 & 0.0363 & 0.0021 & 0.2730 & 0.0101 & 0.3093 & 0.0085 \\
\hline BXD39 & 0.1538 & 0.0294 & 0.1578 & 0.0146 & 0.0249 & 0.0019 & 0.3116 & 0.0161 & 0.3366 & 0.0142 \\
\hline BXD40 & 0.1289 & 0.0131 & 0.1558 & 0.0096 & 0.0296 & 0.0044 & 0.2847 & 0.0211 & 0.3143 & 0.0250 \\
\hline BXD42 & 0.1121 & 0.0125 & 0.1151 & 0.0039 & 0.0286 & 0.0018 & 0.2272 & 0.0099 & 0.2558 & 0.0094 \\
\hline
\end{tabular}


the variance of all measurements (the total phenotypic variance). Calculation was performed with $\mathrm{R}$.

\section{RESULTS \\ MOUSE STRAINS C57BL/6J AND DBA/2J DIFFER IN FIELD SIZES OF THE INFRAPYRAMIDAL MOSSY FIBER TRACT}

Previous studies have shown that mouse strains C57BL/6J and $\mathrm{DBA} / 2 \mathrm{~J}$ differ in the proliferation of precursor cells in the dentate gyrus, the survival of newborn cells, and the net production of new granule cells (Kempermann and Gage, 2002; Kempermann et al., 2006). C57BL/6J mice have more adult hippocampal neurogenesis than DBA/2J mice. Comparisons of the size of the MF subfields, visualized by an immunohistochemical staining of the synaptic vesicle protein synaptoporin (Singec et al., 2002), revealed that in $\mathrm{C} 57 \mathrm{BL} / 6 \mathrm{~J}$ the IMF was twice as big as in DBA/2J animals and that C57BL/6J also had a larger hilus (Figure 2; Table 1). Natural variation in the IMF is thought to be associated with differences in spatial learning (Crusio et al., 1987; Schopke et al., 1991). We had previously tested the same strains of mice in the Morris water maze and had found a better performance of C57BL/6J compared to DBA/2J mice in acquisition and retention of spatial memory (Kempermann and Gage, 2002). In summary, the new and previous data indicate that $\mathrm{C} 57 \mathrm{BL} / 6 \mathrm{~J}$ and DBA/2J differed significantly regarding adult hippocampal neurogenesis, learning of a hippocampus dependent task, and MF distribution.

\section{BXD STRAINS REVEAL LARGE NATURAL VARIATION IN THE MOSSY FIBER TRACT}

To extend the evidence of this relationship we used the set of RI strains of mice BXD that is derived from inbred F2 progeny of parental strains C57BL/6J and DBA/2J. The MF tract was visualized in 31 BXD strains (including parentals), again using synaptoporin immunohistochemistry (Figure 2; Singec et al., 2002). The volume of the MF connection with its three subfields hilus, SMF, and IMF was measured using the Cavalieri estimator on a semi-automated analysis system. Table 1 summarizes the results giving the mean and SE. Bar graphs in Figure 3 visualize the large natural variation of the medians for the same traits by presenting them in ranked order. The variability suggests that these traits are all truly quantitative and polygenic in that the values do not show a bimodal distribution, as would be the case for Mendelian traits. The rank orders are different for the subtraits. Note that the parental strains $\mathrm{C} 57 \mathrm{BL} / 6$ and DBA/2 are not the extremes.

We next explored the impression that the natural variation of the different subtraits (SMF, IMF, etc.) differed between the strains of the BXD panel. Based on our results so far, we expected that IMF would show greater variability than the rest because it is more plastic (Lipp et al., 1988; Schopke et al., 1991). The question was, whether that greater plasticity would still take place on a rather homogenous and narrowly defined genetic background. We found that the size of the SMF correlated well with the total mossy fiber volume (MF) and reasonably with the hilar volume but poorly with the IMF (Table 2). Conversely, the IMF also had low correlation with the hilus and MF.

When the values were correlated with adult hippocampal neurogenesis traits [as deposited in the genenetwork.org database (Kempermann et al., 2006) (accession numbers 10795, 10796, and

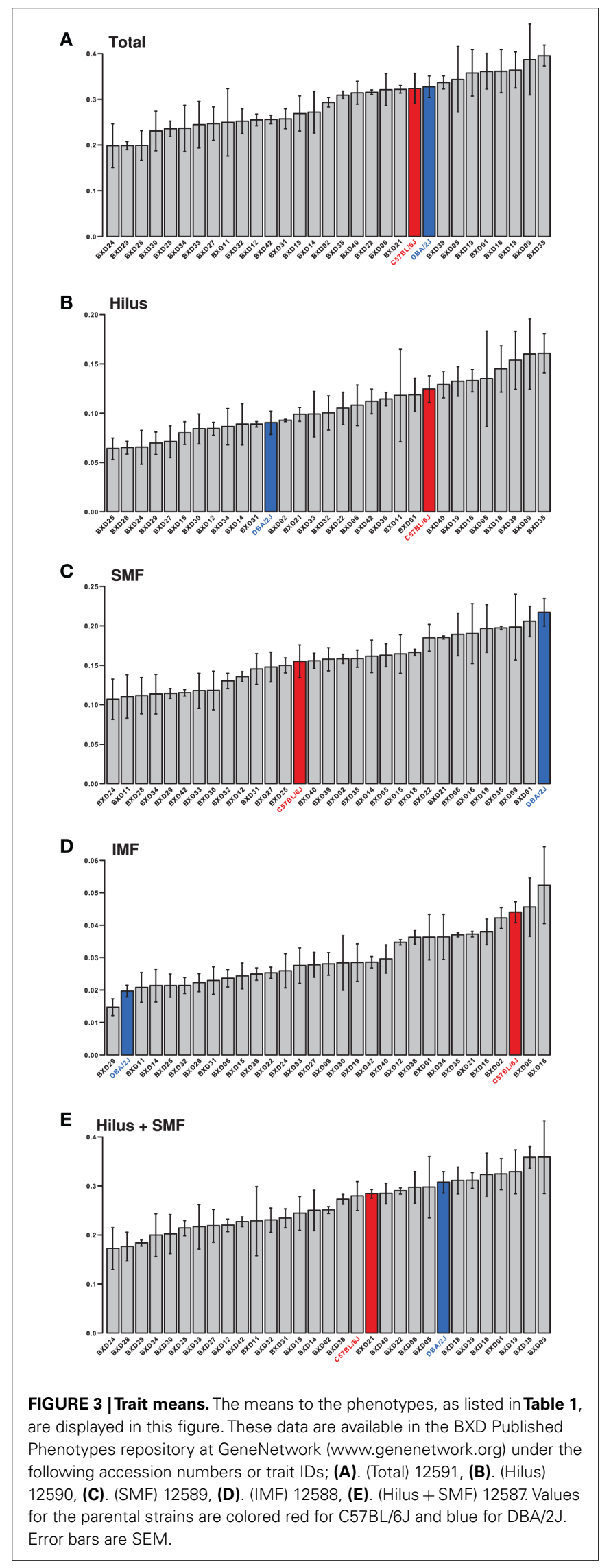


Table 2 | Correlations between measured subtraits.

\begin{tabular}{|c|c|c|c|c|c|c|c|}
\hline & MF (total) & Hilus & IMF & SMF & Prolif. & Survival & New neurons \\
\hline \multicolumn{8}{|c|}{ MOSSY FIBERS } \\
\hline MF (total) & & $0.851 * *$ & 0.510 & $0.881 * *$ & 0.122 & 0.266 & 0.213 \\
\hline IMF & 0.529 & 0.479 & & 0.287 & -0.208 & 0.098 & 0.216 \\
\hline SMF & $0.863 * *$ & 0.516 & 0.240 & & 0.277 & 0.277 & 0.226 \\
\hline Survival & 0.241 & 0.192 & 0.046 & 0.244 & 0.761 & & 0.933 \\
\hline New neurons & 0.235 & 0.147 & 0.150 & 0.245 & 0.741 & 0.947 & \\
\hline
\end{tabular}

The upper diagonal half of the table gives Spearman Rank Correlations, the lower half Pearson's $R$. Associations greater than 0.75 are highlighted in red, those between 0.5 and 0.75 in green.

Table 3 | Heritability of the mossy fiber traits.

\begin{tabular}{llll}
\hline Trait & VG & VP & $\boldsymbol{h}^{\mathbf{2}}$ \\
\hline MF (total) & $3.242 \times 10^{-3}$ & $6.202 \times 10^{-3}$ & 0.52 \\
Hilus & $7.996 \times 10^{-4}$ & $1.564 \times 10^{-3}$ & 0.51 \\
IMF & $7.716 \times 10^{-5}$ & $1.152 \times 10^{-4}$ & 0.67 \\
SMF & $1.051 \times 10^{-3}$ & $2.03 \times 10^{-3}$ & 0.52 \\
\hline
\end{tabular}

10797)], no cross-correlations between the two clusters emerged. This implies that the two plastic systems are genetically to a large degree independent.

\section{IMF HAS A GREATER HERITABILITY THAN THE OTHER SUBFIELDS}

We next calculated the heritability $\left(h^{2}\right)$ of the MF traits. Heritability is the amount of variation of the phenotype in a population that is explained by genetic variation. Heritability of 1 would indicate a completely predictable trait. We have previously published heritability of the "new neurons" trait with $h^{2}=0.70 \pm 0.05$ (Kempermann et al., 2006). We now calculated the heritability of our new traits (Table 3). We found that heritability of IMF was greater than for SMF and hilus but lower than for adult neurogenesis.

Broad-sense heritability of the traits was calculated as the variance of the strain means (the genetic variance) divided by the variance of all measurements (the total phenotypic variance). VG is the variance of strain means and VP is the variance of phenotype. This shows that the IMF trait has in fact a greater heritability $\left(h^{2}\right)$ than the others.

\section{COMPARING QTL MAPPING FOR ADULT HIPPOCAMPAL NEUROGENESIS AND MOSSY FIBER TRAITS}

We next used the mapping tools at www.genenetwork.org to compare the distribution and patterns of potential quantitative trait loci (QTL) for the MF traits compared to adult neurogenesis. As the underlying genomic database was updated since our first study had been published we remapped the published "new neurons" phenotype (Trait ID 10797) for this purpose. The distribution of peaks was unaffected but the calculated LRS scores are slightly lower now. Figure 4 aligns the resulting maps. Reference lines are drawn through the main peaks in the adult neurogenesis trait.
Despite the lack of an overt correlation between adult neurogenesis traits and MF parameters we saw an apparent alignment between some of the peaks in IMF (but not the other traits) and net neurogenesis. Upon closer inspection, the loci for both traits on Chr1 and ChrX were slightly adjacent to each other and thus independent.

We remapped the candidate region on Chromosome 5 at greater resolution and found the overlapping region to lie between 118.3 and 119.2 Mb (Figure 5). This region contains 7 known genes: Nos1, Fbxo21, Tesc, Fbxw8, Hrk, 2410131K14Rik, and Med13l. Of note, the same region is also highlighted in the comparison of IMF with the proliferation phenotype (Trait ID 10795). We now made use of our previously assembled database of hippocampal transcriptome data from the BXD panel (Overall et al., 2009). We mapped the expression QTLs (eQTL) for the candidate genes, except for Med13l (Mediator complex subunit 13-like), which is not present in the gene expression data base, and found that Nos1 (probe set 1438483_at) had a highly significant cis-QTL at the very same location. A cis-QTL means that the expression of the gene maps to its own physical location on the genome. Cis-acting genes are thought to exert strong influences on the traits they are associated with because they are largely autoregulatory and thus less dependent on the activity of other genes.

Knockout mice for Nos1 were reported to show a striking loss in IMF projections (Keilhoff et al., 2001) but have increased cell proliferation and neurogenesis (Zhu et al., 2006; Fritzen et al., 2007).

RIKEN cDNA $2410131 \mathrm{~K} 14$ gene also was also cis-acting, but with lower LRS score (23.0 vs. 36.5 ) and a lower expression in the hippocampus than Nos1. There is no further information on this gene.

\section{DISCUSSION}

In this study we show that the two types of structural plasticity in the hippocampal dentate gyrus, the generation of newborn neurons and the IMF, show a largely independent genetic control. As demonstrated in part 1 of this study, the axons of newborn neurons also contribute to the most plastic part of the MF tract the IMF but not exclusively so.

We reasoned that a straightforward way to explore any partial causative link would be through potentially shared genetics. 


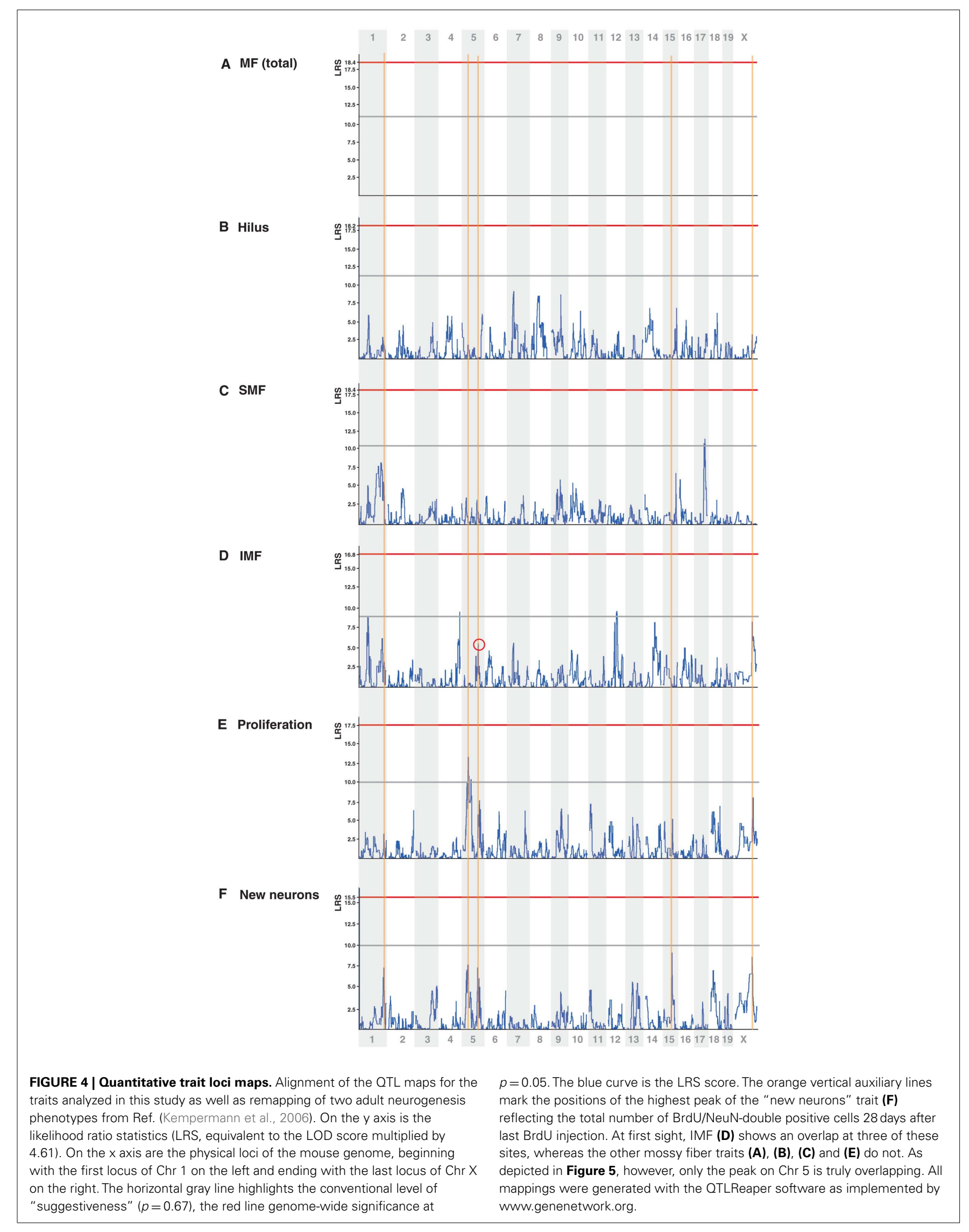




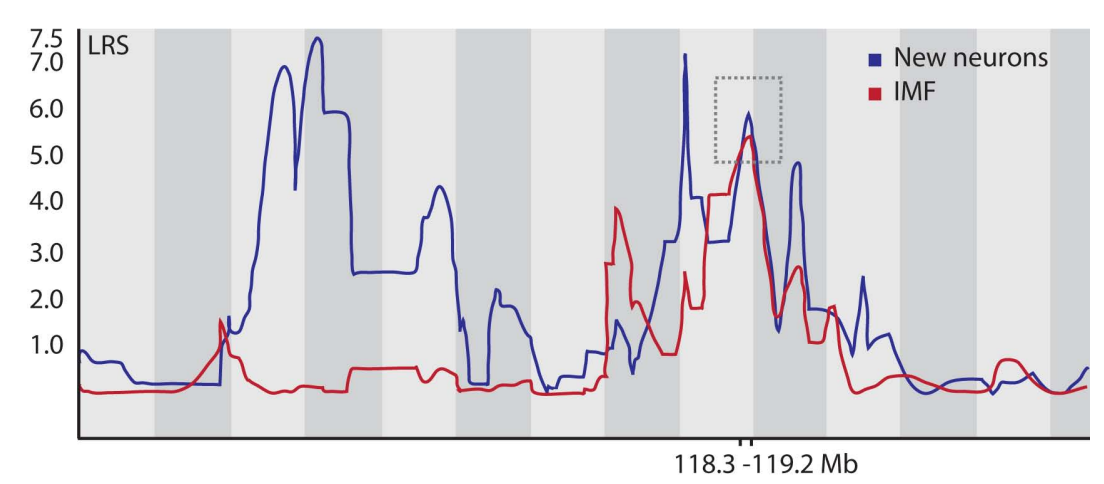

FIGURE 5 | Finemapping on Chr 5. The highest overlapping peak for both the New Neurons (from Ref. Kempermann et al., 2006) and the IMF trait is on distal Chr 5 (marked by the dashed box). The interval spans between 118.3 and 119.2 Megabases. The map was generated with WebQTL (www.genenetwork.org) but redrawn for better graphic resolution. We used 2000 bootstraps for both maps to calculate confidence limits for the QTL and saw sharp peaks in the bootstrap samples, confirming the statistical robustness of the association (details not shown).

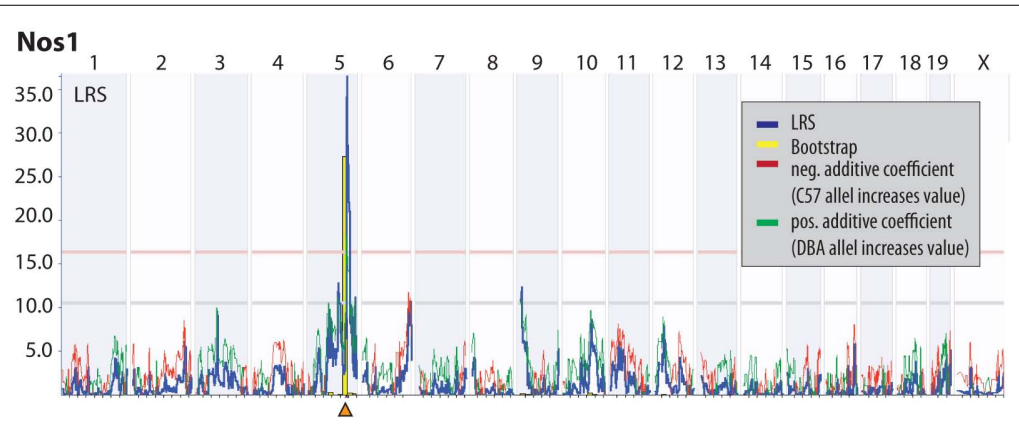

FIGURE 6 | Expression QTL (eQTL) for Nos1. The physical locus of Nos1 is within the interval identified in Figure 5. Nos1 is the only cis-QTLs (i.e., a gene that maps to its own physical location, highlighted by the orange triangle) within this interval.

The underlying rationale was that a shared genetic component explaining a notable part of the variance of both traits would establish a meaningful link in the sense of a common genetic causality.

Our hypothesis was thus that the genetic determinants underlying IMF plasticity might be closely linked to the genetic network controlling adult neurogenesis (Kempermann et al., 2006). But when we assessed adult hippocampal neurogenesis and the size of the IMF in RI mice of the BXD panel - derived by inbreeding of $\mathrm{F} 2$ progeny from the inbred mouse strains $\mathrm{C} 57 \mathrm{BL} / 6 \mathrm{~J}$ and $\mathrm{DBA} / 2 \mathrm{~J}$ - we found little indication of a genetic link. Interestingly, IMF also showed little genetic similarity to the other MF subfields. The size of the MF tract was found to exhibit a relatively moderate variance across the strains of the panel, but this variance was shown to be highly heritable. The high heritability demonstrates a strong genetic source of the variability and suggests that the genetic causes underlying this trait are amenible to mapping in the BXD panel.

We identified the highest QTL peak that was shared by adult neurogenesis and any of the MF traits. This common QTL between "new neurons" and IMF lies between 118.3 and $119.2 \mathrm{Mb}$ on distal Chr 5 and has an LRS score of approximately 5. By all standards this is a very low value, indicating that this locus contributes to only a small fraction of the multigenic regulation of IMF volume. Within this locus, we discovered that the most promising candidate gene, Nos1 (Figure 6), according to the literature should decrease adult neurogenesis but increase IMF size. In concordance with this, we found that, for both Nos1 and IMF volume, the DBA/2J allele increases the trait value, whereas for adult neurogenesis the C57BL/6J allele increases the trait value. According to the literature, Nos1 has opposite effects on both traits (Keilhoff et al., 2001; Packer et al., 2003; Zhu et al., 2006; Fritzen et al., 2007). A role of Nos1 in network refinement through axonal pruning is welldescribed for the visual system (Mize and Lo, 2000) and might be applicable here, too. Nitric oxide was also shown to decrease neurogenesis from cortical progenitor cells in the fetal rodent brain (Cheng et al., 2003). In summary, Nos1 expression is associated positively with levels of adult neurogenesis and negatively with IMF volume, and suggests a potential functional link between the genetic regulation of the two traits.

Our mapping study yielded different results than a previous study from Lassalle et al. (1999), who had also used BXD mice to explore the genetic determinants of the size of the MF fields. In their study $\mathrm{N}$ was 28 strains, in ours 31 strains 
with an overlap of was 24 . These trait values are also available at www.genenetwork.org. Both studies agree on the conclusion, however, that the MF connectivity is under strong genetic control. We estimated heritability for the total MF volume to be 0.44 , Lassalle et al. (1999) (for the corresponding area) 0.36. Values for the infrapyramidal projection were even higher. Despite the very limited mapping power at the time, Lassalle et al. (1999) proposed a number of preliminary QTL, mostly for derivative, relative traits (e.g., hilar area as percentage of the total area). The study might be challenged on the ground of the methods used to assess the size of the MF projection, which treated the fiber tract essentially as two-dimensional structure and not as volume. Consequently, there is otherwise little consistency between the two studies.

In a large di-allelic F1 study between five commonly used laboratory strains of mice, Crusio et al. (2007) had found that the only hippocampal subregion for whose size additive genetic effects and a directional dominance for high values was found, was the suprapyramidal mossy fiber projection. They argue that this observation would speak in favor of an evolutionary pressure toward larger suprapyramidal projections.

Genetic variation in the MF projection with relatively stable contribution of the SMF is also found in other species, as shown in a study on two vole species that also showed a correlation between

\section{REFERENCES}

Bernasconi-Guastalla, S., Wolfer, D. P., and Lipp, H. P. (1994). Hippocampal mossy fibers and swimming navigation in mice: correlations with size and left-right asymmetries. Hippocampus 4, 53-63.

Blackstad, T. W., Brink, K., Hem, J., and Jeune, B. (1970). Distribution of hippocampal mossy fibers in the rat. An experimental study with silver impregnation methods. J. Comp. Neurol. 138, 433-449.

Cheng, A., Wang, S., Cai, J., Rao, M. S., and Mattson, M. P. (2003). Nitric oxide acts in a positive feedback loop with BDNF to regulate neural progenitor cell proliferation and differentiation in the mammalian brain. Dev. Biol. 258, 319-333.

Crusio, W. E., Genthner-Grimm, G., and Schwegler, H. (2007). A quantitativegenetic analysis of hippocampal variation in the mouse. J. Neurogenet. 21, 197-208.

Crusio, W. E., and Schwegler, H. (2005). Learning spatial orientation tasks in the radial-maze and structural variation in the hippocampus in inbred mice. Behav. Brain Funct. 1, 3.

Crusio, W. E., Schwegler, H., and Lipp, H. P. (1987). Radial-maze performance and structural variation of the hippocampus in mice: a correlation with mossy fibre distribution. Brain Res. 425, 182-185.

Crusio, W. E., Schwegler, H., and van Abeelen, J. H. (1989). Behavioral

maze performance and plasticity of the IMF (Pleskacheva et al., 2000).

In summary, we identified interesting but not fully conclusive links between adult hippocampal neurogenesis and the plasticity of the IMF. The previously reported, highly suggestive coregulation was not matched by a shared genetic control. Nevertheless, we found both traits to be highly heritable and could pinpoint one shared candidate locus that, however, might exert only a very small overall effect on both traits, and which contains a very interesting cis-QTL and candidate gene, Nos1. Besides the fact that the genetic independence between IMF and the other MF traits and adult neurogenesis is remarkable in its own right, our study also cautions how deceiving phenotypic correlations might be in terms of the supposedly underlying genetics.

\section{ACKNOWLEDGMENTS}

This work was supported by VolkswagenStiftung (to Gerd Kempermann) and NCCR Neural Plasticity and Repair (to Sebastian Jessberger). We would also like to thank Irene Thun, Ruth Zarmstorff, and Silke Kurths for technical support. Benedikt Römer was supported by GRK 238, Deutsche Forschungsgemeinschaft. Julia Krebs was an associate fellow of International Max Planck Research School LIFE.

Laghmouch, A., Bertholet, J. Y., and Crusio, W. E. (1997). Hippocampal morphology and open-field behavior in Mus musculus domesticus and Mus spretus inbred mice. Behav. Genet. 27, 67-73.

Lassalle, J. M., Halley, H., Milhaud, J. M., and Roullet, P. (1999). Genetic architecture of the hippocampal mossy fiber subfields in the BXD RI mouse strain series: a preliminary QTL analysis. Behav. Genet. 29, 273-282.

Lipp, H. P., Schwegler, H., and Driscoll, P. (1984). Postnatal modification of hippocampal circuitry alters avoidance learning in adult rats. Science 225, 80-82.

Lipp, H. P., Schwegler, H., Heimrich, B. and Driscoll, P. (1988). Infrapyramidal mossy fibers and two-way avoidance learning: developmental modification of hippocampal circuitry and adult behavior of rats and mice. J. Neurosci. 8, 1905-1921.

Mize, R. R., and Lo, F. (2000). Nitric oxide, impulse activity, and neurotrophins in visual system development(1). Brain Res. 886, 15-32.

Nowakowski, R. S. (1984). The mode of inheritance of a defect in lamination in the hippocampus of BALB/c mice. J. Neurogenet. 1, 249-258.

Overall, R. W., Kempermann, G., Peirce, J., Lu, L., Goldowitz, D., Gage, F. H., Goodwin, S., Smit, A. B., Airey, D. C., Rosen, G. D., Schalkwyk, L. C., Sutter, T. R., Nowakowski, R. S., Whatley, S., and Williams, R. W. (2009).
Genetics of the hippocampal transcriptome in mouse: a systematic survey and online neurogenomics resource. Front. Neurosci. 3:55. doi: 10.3389/neuro.15.003.2009

Packer, M. A., Stasiv, Y., Benraiss, A., Chmielnicki, E., Grinberg, A., Westphal, H., Goldman, S. A., and Enikolopov, G. (2003). Nitric oxide negatively regulates mammalian adult neurogenesis. Proc. Natl. Acad. Sci. U.S.A. 100, 9566-9571

Pleskacheva, M. G., Wolfer, D. P., Kupriyanova, I. F., Nikolenko, D. L., Scheffrahn, H., Dell'Omo, G., and Lipp, H. P. (2000). Hippocampal mossy fibers and swimming navigation learning in two vole species occupying different habitats. Hippocampus 10 17-30.

Römer, B., Krebs, J., Overall, R., Fabel, K., Babu, H., Overstreet Wadiche, L., Brandt, M., Williams, R. W., Jessberger, S., and Kempermann, G. (2011). Adult hippocampal neurogenesis and plasticity in the infrapyramidal bundle of the mossy fiber projection: I. Coregulation by activity. Front. $\mathrm{Neu}$ rosci. 5:107. doi: 10.3389/fnins.2011. 00107

Roullet, P., and Lassalle, J. M. (1990). Genetic variation, hippocampal mossy fibres distribution, novelty reactions and spatial representation in mice. Behav. Brain Res. 41, 61-70. 
Schopke, R., Wolfer, D. P., Lipp, H. P., and Leisinger-Trigona, M. C. (1991). Swimming navigation and structural variations of the infrapyramidal mossy fibers in the hippocampus of the mouse. Hippocampus 1 , 315-328.

Schwegler, H., Crusio, W. E., and Brust, I. (1990). Hippocampal mossy fibers and radial-maze learning in the mouse: a correlation with spatial working memory but not with non-spatial reference memory. Neuroscience 34 , 293-298.

Schwegler, H., Crusio, W. E., Lipp, H. P., and Heimrich, B. (1988). Watermaze learning in the mouse correlates with variation in hippocampal morphology. Behav. Genet. 18, 153-165.

Schwegler, H., Lipp, H. P., Van der Loos, H., and Buselmaier, W. (1981). Individual hippocampal mossy fiber distribution in mice correlates with two-way avoidance performance. Science 214, 817-819.
Shipley, B. (2002). Cause and Correlation in Biology: A User's Guide to Path Analysis, Structural Equations and Causal Inference. Cambridge: Cambridge University Press.

Singec, I., Knoth, R., Ditter, M., Hagemeyer, C. E., Rosenbrock, H., Frotscher, M., and Volk, B. (2002). Synaptic vesicle protein synaptoporin is differently expressed by subpopulations of mouse hippocampal neurons. J. Comp. Neurol. 452, 139-153.

Taylor, B. A. (1978). "Recombinant inbred strains: use in gene mapping," in Origins of Inbred Mice, ed. H. Morse (New York, NY: Academic Press), 423-438.

Taylor, B. A., Wnek, C., Kotlus, B. S., Roemer, N., MacTaggart, T., and Phillips, S. J. (1999). Genotyping new BXD recombinant inbred mouse strains and comparison of BXD and consensus mapps. Mamm. Genome 10, 335-348.

Toni, N., Laplagne, D. A., Zhao, C., Lombardi, G., Ribak, C. E., Gage, F. H., and Schinder, A. F. (2008). Neurons born in the adult dentate gyrus form functional synapses with target cells. Nat. Neurosci. 11, 901-907.

Zhao, C., Teng, E. M., Summers, R. G. Jr., Ming, G. L., and Gage, F. H. (2006). Distinct morphological stages of dentate granule neuron maturation in the adult mouse hippocampus. J. Neurosci. 26, 3-11.

Zhu, X. J., Hua, Y., Jiang, J., Zhou, Q. G., Luo, C. X., Han, X., Lu, Y. M., and Zhu, D. Y. (2006). Neuronal nitric oxide synthasederived nitric oxide inhibits neurogenesis in the adult dentate gyrus by down-regulating cyclic AMP response element binding protein phosphorylation. Neuroscience 141, 827-836.

Conflict of Interest Statement: The authors declare that the research was conducted in the absence of any commercial or financial relationships that could be construed as a potential conflict of interest.
Received: 06 May 2011; paper pending published: 25 July 2011; accepted: 29 August 2011; published online: 21 September 2011.

Citation: Krebs J, Römer B, Overall $R W$, Fabel K, Babu H, Brandt MD, Williams RW, Jessberger $S$ and Kempermann G (2011) Adult hippocampal neurogenesis and plasticity in the infrapyramidal bundle of the mossy fiber projection: II. Genetic covariation and identification of Nos1 as linking candidate gene. Front. Neurosci. 5:106 doi: 10.3389/fnins.2011.00106

This article was submitted to Frontiers in Neurogenesis, a specialty of Frontiers in Neuroscience.

Copyright (๑ 2011 Krebs, Römer, Overall, Fabel, Babu, Brandt, Williams, Jessberger and Kempermann. This is an open-access article subject to a nonexclusive license between the authors and Frontiers Media SA, which permits use, distribution and reproduction in other forums, provided the original authors and source are credited and other Frontiers conditions are complied with. 